\title{
Oxidative damage to DNA: Inhibition of guanine damage* $^{*}$
}

\author{
Sriram Kanvah and Gary B. Schuster $\ddagger$ \\ School of Chemistry and Biochemistry, Georgia Institute of Technology, Atlanta, GA \\ 30332, USA
}

\begin{abstract}
One-electron oxidation of DNA results in chemical damage to nucleobases, particularly guanine in multiple $\mathrm{G}$ sequences. Oxidation may be triggered by numerous events, including photosensitization. We describe studies of photoinduced oxidations of DNA triggered by irradiation of covalently linked anthraquinone derivatives under various conditions that affect the global structure of the DNA. These structural changes have subtle effects on the result of the one-electron oxidation.
\end{abstract}

Keywords: oxidative damage; guanine damage; DNA; molecular wire; nanoelectronics; damage repair.

The examination of electron-transfer reactions in DNA has attracted attention for two different but related reasons. First, the apparently well-stacked $\pi$-electron array of nucleobases in DNA has stimulated interest in its electronic properties and has led to speculation that DNA has potential uses as a "molecular wire" in nanoelectronics [1]. Second, the removal of an electron from DNA has been shown to cause reaction to occur at locations that are far away from the site of the initial oxidation. These reactions generate mutated DNA bases, which can have biological consequences [2].

Oxidative damage to DNA can be initiated by UV light, ionizing radiation, or normal metabolic processes that result in removal of an electron with the concomitant formation of a radical cation ("hole"). The radical cation may migrate through the DNA by hopping until it is trapped by an irreversible reaction with $\mathrm{H}_{2} \mathrm{O}$ or $\mathrm{O}_{2}$ to form 8-oxo-7, 8-dihydroguanine (8-oxoG) [3], and other oxidation products. Consequently, it is important that living systems protect DNA from reactions caused by electron loss. Damage repair mechanisms have evolved as one strategy. An alternative strategy would be to drive reactive radical cations away from critical coding regions of genomic DNA. In this regard, it has been suggested that DNA itself may contain sacrificial nucleobase sequences in introns that are positioned optimally to absorb holes and thereby protect the bases in adjacent exons from oxidative damage [4]. Alternately, it may be possible that noncovalently bound sacrificial reagents are able to protect DNA bases from oxidative damage.

\section{PHOTOOXIDATIVE DAMAGE TO DNA}

A variety of photooxidants [5] have been used to initiate reactions that lead to strand cleavage of DNA. We have employed an anthraquinone derivative that is covalently linked to a 5'-end of DNA. Irradiation

\footnotetext{
*Paper based on a presentation at the XXI ${ }^{\text {st }}$ IUPAC Symposium on Photochemistry, 2-7 April 2006, Kyoto, Japan. Other presentations are published in this issue, pp. 2193-2359.

¥Corresponding author
} 
of the covalently linked anthraquinone introduces a radical cation that hops through the DNA causing oxidative damage [6,7]. Subsequent piperidine treatment of the irradiated sample results in strand cleavage at oxidized sites that can be analyzed by gel electrophoresis and visualized by autoradiography.

Among the common DNA nucleobases, guanine has the lowest oxidation potential [8], and, hence, oxidative reactions are commonly found to occur primarily at guanines-especially those that are together in such sequences as a GG step or GGG segment. Less common nucleobases, such as 8-OxoG, can have a much lower oxidation potential than guanine, and they have been found to act as deep traps $[9,19]$ for radical cations. Surprisingly, it has recently been found that one-electron oxidation of DNA sequences that lack guanines leads to reaction at thymines, which has a high oxidation potential [10]. Altogether, the site and extent of reaction at a particular nucleobase following light-induced one-electron oxidation emerges after consideration of the rates of charge hopping and trapping [11] and the binding of ligands and counter ions [7,12].

\section{PROTECTING DNA FROM OXIDATIVE DAMAGE}

Multivalent cations such as spermine and spermidine are known to associate and stabilize DNA oligonucleotides $[13,14]$ and to play an important role in packaging. Both spermine and spermidine bind to DNA nonselectively and occupy sites along the backbone due to strong electrostatic association bridging both the major and minor grooves [15]. We examined the protection of DNA by polyamine derivatives (Fig. 1). We also assessed the effect of 8-oxoG as a low-oxidation-potential "sink" to examine the generality of protection of DNA.<smiles>C[NH2+]CCC[NH2+]CCC[NH2+]CCC[NH3+]</smiles>

Spermine<smiles>[NH3+]CCC[NH2+]CCSSCC[NH2+]CCC[NH3+]</smiles>

Spermine disulfide

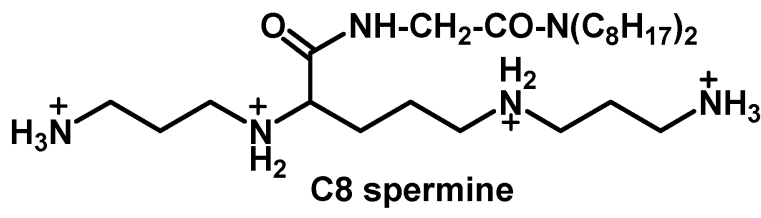<smiles>[NH3+]CCCC[NH2+]CCC[NH3+]</smiles>

Spermidine

Fig. 1 Chemical structures of polyamines utilized in this study. At physiological $\mathrm{pH}$, polyamines are polycationic in nature.

\section{DESIGN OF SEQUENCES}

The DNA oligomers that we investigated are shown in Fig. 2. In the first set, DNA (1-3), there are two GG steps on the complementary (non-AQ-containing) strand while DNA (4) has six GG steps separated 
by AAA bridges and has a AGCT overhang at its 5 '-end to facilitate ligation. In another set, DNA (5) and DNA (6), there are eight GG steps separated by AA bridges, and in a particular case, one of the guanines is replaced by an 8-oxoG. Each duplex has an AQ group that is covalently linked to the $5^{\prime}$ end of one strand, and a ${ }^{32} \mathrm{P}$-radiolabel is incorporated at the 5 '- or 3 '-end (indicated by $*$ in Fig. 2) of the complementary strand. By this construction, we systematically probed the electrostatic effect of polycationic polyamines on DNA charge transport and reaction.

\begin{tabular}{|c|c|}
\hline DNA & \\
\hline 1 & $\begin{array}{r}5^{\prime}-\mathrm{AQ}-\mathrm{AAAGCCAAAACCGTAGAAC}-3^{\prime} \\
\text { TTTCGGTTTTGGCATCTTG } * 5^{\prime}\end{array}$ \\
\hline 2 & $\begin{array}{r}5^{\prime}-\mathrm{AQ}-\mathrm{AAAGCCTTAACCGTAGAAC}-\mathbf{3}^{\prime} \\
\text { TTTCGGAATTGGCATCTTG } *-5^{\prime}\end{array}$ \\
\hline 3 & $\begin{array}{r}5^{\prime}-\mathrm{AQ}-\mathrm{AAATGCCGTACAAACATGGCCGTACGACG}-\mathbf{3}^{\prime} \\
\text { TTTACGGCATGTTTGTACCGGCATGCTGC } *-5^{\prime}\end{array}$ \\
\hline 4 & $\begin{array}{l}5^{\prime} \text {-AGCTGATATCAGGAAAGGAAAGGAAAGGAAAGGAAAGGATAATAATG* }-3^{\prime} \\
3^{\prime}-\quad \text { CTATAGTCCTTTCCTTTCCTTTCCTTTCCTTCCTATTATTAC-AQ-5' }\end{array}$ \\
\hline 5 & 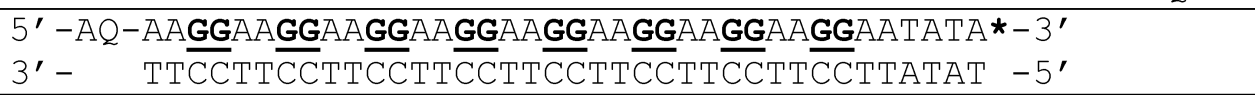 \\
\hline 6 & 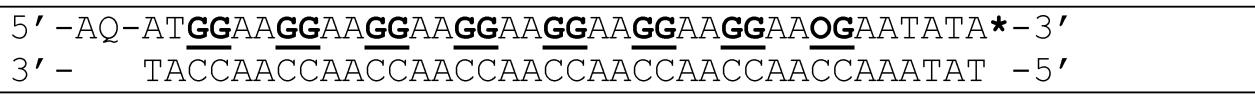 \\
\hline
\end{tabular}<smiles>[B]C1CC(O)C(OS(=O)(=O)OCCNC(=O)c2ccc3c(c2)C(=O)c2ccccc2C3=O)O1</smiles><smiles>Nc1nc2c([nH]c(=O)n2[Ga])c(=O)[nH]1</smiles>

O: (7,8-dihydro 8-oxoguanosine)

Fig. 2 Structure of DNA oligomers. Anthraquinone is covalently linked to $5^{\prime}$-end of the DNA. Radioactive $\mathrm{P}^{32}$ (* in the figure) is used to tag one end of the DNA to visualize using autoradiography and polyacrylamide gel electrophoresis. 8-OxoG replaces one of the guanines in the GG step in DNA (6).

\section{EFFECT OF POLYAMINE DERIVATIVES ON DNA STRUCTURE}

The addition of spermine to solutions of DNA (1-3) causes the melting temperature $\left(T_{\mathrm{m}}\right)$ of the DNA to increase $\left(\Delta T \sim 14{ }^{\circ} \mathrm{C}\right)$, which indicates that spermine associates with the DNA and stabilizes it, while addition of the $\mathrm{C}_{8}$-spermine derivative leads to a smaller melting temperature increase $\left(\Delta T \sim 4{ }^{\circ} \mathrm{C}\right)$. Addition of spermine disulfide to DNA $(\mathbf{1 , 2})$ also yields similar results with a concomitant increase of $T_{\mathrm{m}}$, but the magnitude of the increase is smaller than that for spermine $\left(\Delta T \sim 12{ }^{\circ} \mathrm{C}\right.$, Fig. 3$)$ The addition of spermine or spermine disulfide did not significantly affect the circular dichroism spectra (CD) of DNA (1-3). However, addition of high concentrations of the $\mathrm{C}_{8}$-spermine derivative results in minor changes to the $\mathrm{CD}$ spectrum, which may indicate formation of secondary structures due to wrapping of the alkyl chains along the major and minor grooves. 


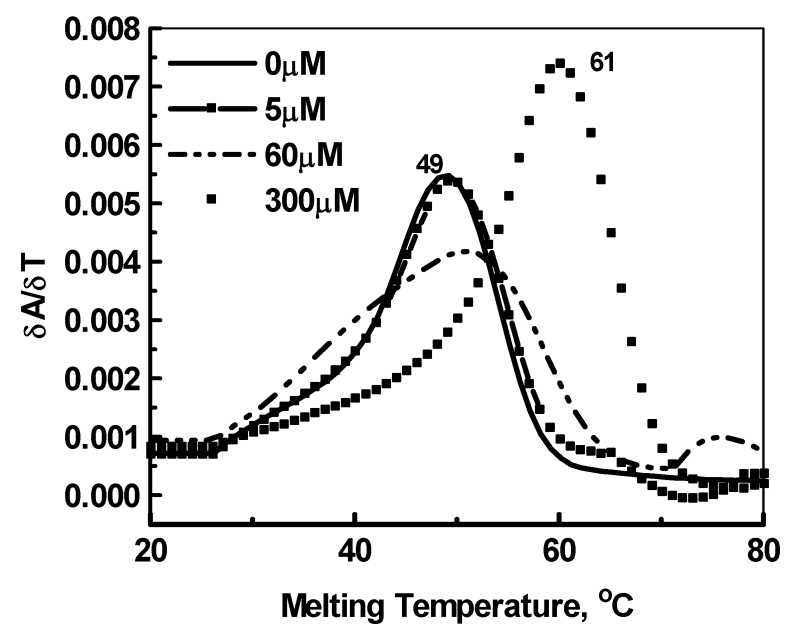

Fig. 3 Melting curves for DNA (2) with increased addition of spermine disulfide. The melting curves are presented as first derivative plots and are obtained in standard phosphate buffer solution containing $2.0 \mu \mathrm{M}$ of the duplex and monitored at $260 \mathrm{~nm}$.

\section{INHIBITION OF DNA DAMAGE: DNA-SPERMINE DISULFIDE ASSOCIATION}

A radiolabeled sample of duplex DNA (1, 2, or 3) was irradiated using 350-nm light and then subsequently treated with piperidine. The irradiated samples were analyzed by denaturing gel electrophoresis and visualized by autoradiography. The results obtained for DNA (1) are shown in Fig. 4.

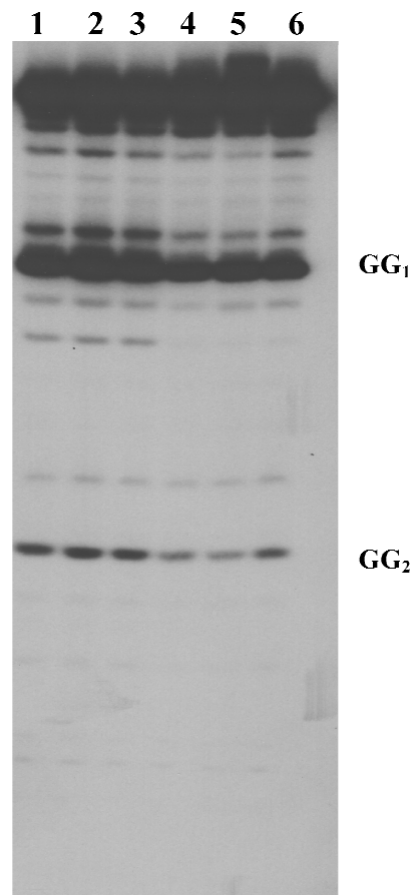

Fig. 4 Autoradiogram obtained from the irradiation of DNA (1) in the presence of various concentrations of spermine disulfide. Lanes 1-6 corresponds to $0,2.5,5,25,50,300 \mu \mathrm{M}$ concentrations of spermine disulfide. Increased addition of spermine disulfide leads to inhibited guanine damage. 
Irradiation causes strand cleavage primarily at the 5'-G of the GG steps of these oligomers. The GG step $\left(\mathrm{GG}_{1}\right)$ that is closer to $\mathrm{AQ}$ has a greater amount of damage than $\left(\mathrm{GG}_{2}\right)$, which is farther away. The ratio of strand cleavage for $\mathrm{GG}_{1} / \mathrm{GG}_{2}(\sim 12)$ in DNA(2) is not measurably affected by addition of up to $300 \mu \mathrm{M}$ of spermine (lanes 6-10). In contrast, addition of the spermine disulfide leads to a threefold increase in the ratio of reaction of $\mathrm{GG}_{2}$ to $\mathrm{GG}_{1}$. This finding shows that transfer of an electron from the bound disulfide group to a nucleobase radical cation competes effectively with the migration of the charge and its reaction with water, leading to protection of DNA from oxidative damage [15].

\section{DNA-SPERMINE LIPID-LIKE COMPLEXES}

The pattern of reactivity in DNA oligomers that have multiple GG steps has been shown to be controlled by the relative magnitudes of the rates for the hopping of the radical cation $\left(k_{\text {hop }}\right)$ from one GG step to another and for the trapping reaction of the radical cation $\left(k_{\text {trap }}\right)$ with $\mathrm{H}_{2} \mathrm{O}$ or $\mathrm{O}_{2}$. Thus, the ratio $k_{\text {hop }} / k_{\text {trap }}$ determines the observed pattern of reactivity in DNA oligomers containing multiple GG steps.

DNA (3) has two GG steps on the strand complementary to the one linked to the anthraquinone charge injector. As is often the case, GG steps that are closer to the AQ react more efficiently than those that are farther away. Addition of the $\mathrm{C}_{8}$-spermine derivative to the DNA solution before irradiation yields results that are distinctly different than the effect observed for addition of spermine (Fig. 5). The addition of the $\mathrm{C}_{8}$-spermine causes a two-fold decrease in the relative reactivity at the GG steps [16].

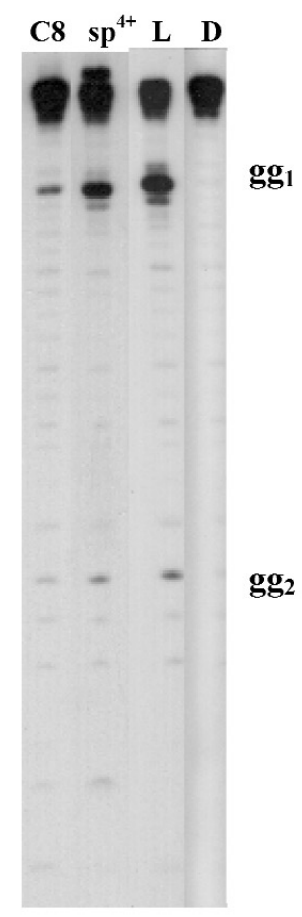

Fig. 5 Autoradiogram from the irradiation of DNA (3) in the presence of 2 equiv of spermine and C8 spermine. L is light control for DNA (3) without addition of spermine or C8-spermine, and D is the un-irradiated dark control. All the samples have been irradiated for $3 \mathrm{~min}$ and subsequently treated with $1 \mathrm{M}$ piperidine before gel electrophoresis. 
Due to their polycationic nature, the $\mathrm{C}_{8}$-spermine and spermine are expected to bind in a similar fashion. The presence of long alkyl chains in the $\mathrm{C}_{8}$-spermine allows wrapping of its hydrophobic alkyl chains around the major and minor grooves of the DNA leading to formation of a hydrophobic surface layer. This coating is proposed to effectively reduce $k_{\text {trap }}$ by impeding the ability of polar reagents such as $\mathrm{H}_{2} \mathrm{O}$ to penetrate to the DNA and react with a nucleobase radical cation. This reduced rate for the reaction with water for DNA-spermine complex accounts for the protective effect. The distance dependence of the migration process becomes weaker, and the efficiency of strand cleavage is reduced [16].

\section{MIMICKING DNA IN CELLS: DNA CONDENSATES}

Most studies of oxidative damage to DNA have focused on one-electron oxidation of oligomers in solution. However, DNA in cells is tightly packed and does not closely resemble oligonucleotides. Migration of a radical cation and its reaction in DNA condensates provides an approximation of the behavior of oxidized DNA in cells. To assess the effect of condensate formation on long-distance radical cation hopping, linearized pUC19 plasmid was ligated to the oligonucleotide DNA (4), which contains a series of GG steps and a covalently linked anthraquinone group. Treatment of the ligated plasmid with spermidine results in formation of condensates. These condensates were detected by dynamic lightscattering experiments and by transmission electron microscopy [17]. Irradiation of the anthraquinone group in the condensate initiates a one-electron oxidation injecting a radical cation that migrates by a hopping mechanism causing a chemical reaction with $\mathrm{H}_{2} \mathrm{O}$ or $\mathrm{O}_{2}$ leading to a strand cleavage at GG steps in the ligated oligonucleotide. The efficiency of charge migration in the condensate is reduced compared with that of the corresponding oligomer in solution; Fig. 6 shows the results [17].

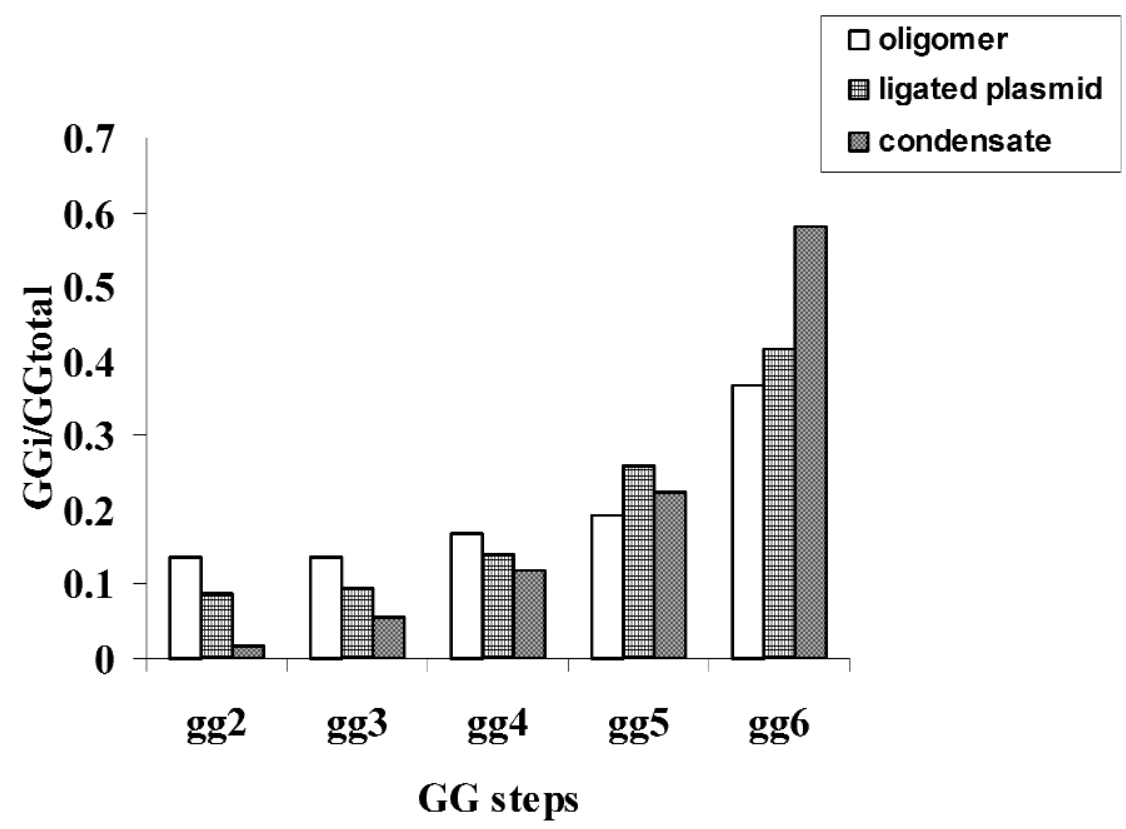

Fig. 6 Histograms showing the relative amount of reaction at GG sites for DNA (4) obtained after 10 min of irradiation. Oligomer is the control that is not ligated and was not treated with spermidine. Ligated plasmid is the oligomer that is ligated to plasmid but not condensed. 


\section{PROTECTIVE EFFECT OF 8-OXOG ON THE REACTION AND TRANSPORT}

8-OxoG has an oxidation potential that is $\sim 0.4 \mathrm{~V}$ below that of guanine and is more susceptible to oxidative reactions than the normal nucleobases. DNA (5) and DNA (6) have eight GG steps separated by AA bridges. An 8-oxoG is strategically placed, replacing a 5'-G of the eighth GG step in DNA (6). Irradiation leads to oxidative damage of all GG steps in DNA (5), while in DNA (6) only the 8-oxoG reacts, Fig. 7. In DNA (5) and DNA (6), the charge migration through AA bridges is much faster than is its consumption at a GG step $[18,19]$. However, the radical cation is irreversibly consumed the first time it encounters an 8-oxoG, and thus no reaction is detected at any other nucleobase in the oligomer. Similar protection of guanine nucleobases is obtained when AA bridges are replaced by AT bridges. However, the introduction of a greater barrier to the radical cation migration such as ATTA bridge reduces the protective effect that 8-oxoG has on the preceding GG steps [19].

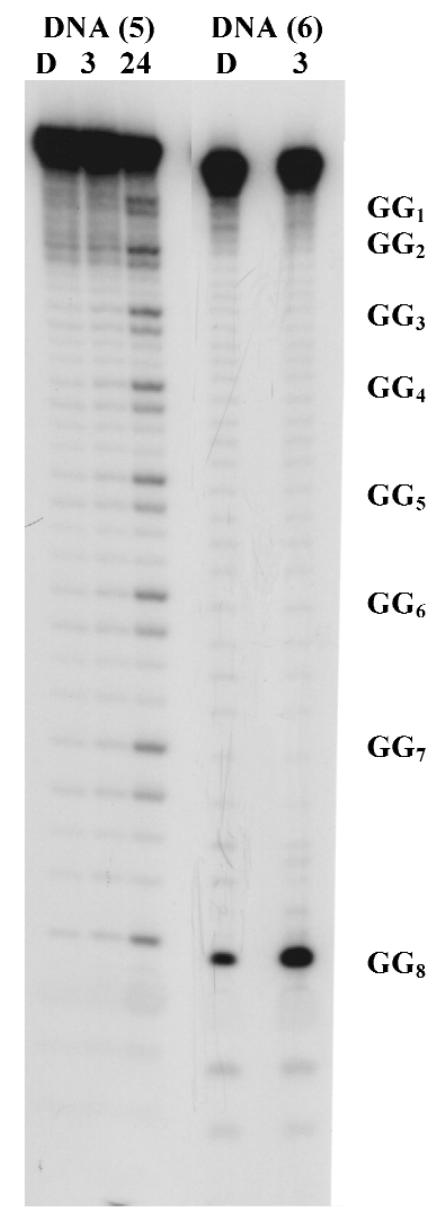

Fig. 7 Autoradiograms from the irradiation of DNA (5) and DNA (6). D is dark control, 3 and 24 are minutes of irradiation. 3 minutes of irradiation for 8-oxoG containing strands [DNA (6)] is sufficient to induce an efficient reaction at 8-oxoG step and subsequent inhibition at GG steps. 


\section{CONCLUSIONS}

It is clear from the experiments reported here that guanines in DNA may be protected from oxidative damage by a number of strategies. Packaging DNA with lipid-like compounds provides some protection [20]. Low-oxidation-potential sacrificial donors such as a disulfide or an 8-oxoG will protect guanines located nearby. And the formation of complex structures such as condensates reduces the amount of oxidative damage observed.

\section{ACKNOWLEDGMENTS}

Financial support from the National Science Foundation (NSF) and the Vasser-Woolley foundation is greatly appreciated. G. B. S. thanks his previous and current students who have contributed to the work on charge migration in DNA. It is because of their hard work this effort has been productive

\section{REFERENCES}

1. R. G. Endres, D. L. Cox, R. R. P. Singh. Rev. Mod. Phys. 76, 195 (2004).

2. H. E. Poulsen, H. Prieme, S. Loft. Eur. J. Cancer Prev. 7, 9 (1998).

3. C. J. Burrows, G. J. Muller. Chem. Rev. 98, 1109 (1998).

4. A. Heller. Faraday Discuss. 116, 1 (2000).

5. B. Armitage. Chem. Rev. 98, 1171 (1998).

6. G. B. Schuster. Acc. Chem. Res. 33, 253 (2000).

7. G. B. Schuster, U. Landman. Top. Curr. Chem. 236, 139 (2004).

8. S. Steenken, S. V. Jovanovic. J. Am. Chem. Soc. 119, 617 (1997).

9. S. M. Gasper, G. B. Schuster. J. Am. Chem. Soc. 119, 12762 (1997).

10. A. Joy, A. Ghosh, G. B. Schuster. J. Am. Chem. Soc. 128, 5346 (2006).

11. J. Joseph, G. B. Schuster. J. Am. Chem. Soc. 128, 6070 (2006).

12. A. Joy. Chem. Commun. 22, 2778 (2005).

13. B. G. Feurstein, N. Pattabhiraman, L. J. Marton. Proc. Natl. Acad. Sci. USA 83, 5948 (1986).

14. M. H. Hou, S. B. Lin, J. M. Yuann, W. C. Lin, A. H. Wang, L.-S. Kan. Nucleic Acids Res. 29, $5121(2001)$.

15. S. Kanvah, G. B. Schuster. J. Am. Chem. Soc. 124, 11286 (2002).

16. H. Cao, G. B. Schuster. Bioconjugate Chem. 16, 820 (2005).

17. P. Das, G. B. Schuster. Proc. Natl. Acad. Sci. USA 102, 14227 (2005).

18. C. S. Liu, G. B. Schuster. J. Am. Chem. Soc. 126, 2877 (2004).

19. S. Kanvah, G. B. Schuster. Nucleic Acids Res. 33, 5133 (2005).

20. C. C. Bjorklund, W. B. Davis. Nucleic Acids Res. 34, 1836 (2006). 